COMMENT. Children with ADHD have significantly prolonged sleep duration and a trend toward a lower percentage of quiet sleep, possibly attributed to hypoarousal or fatigue. Normalization of sleep patterns and decreased sleep duration achieved by methylphenidate could result from increased arousal. Insomnia, frequently reported as a side effect of methylphenidate, requires closer study.

Perceptions of methylphenidate effects on peer interactions of ADHD children were studied by psychologists at the University of California, Los Angeles and Irvine. (Granger DA et al. I Abnormal Child Psychol 1993;21:535). Analyses of observations of videotapes by 96 undergraduates showed that medication increased social withdrawal and dysphoria/disengagement, suggesting negative interpersonal consequences of these unintended internalizing behavior changes, even when not cued by rating scales. A positive medication effect was obtained in the category of leader/planner, behaviors requiring social organization and foresight.

\title{
ASPARTAME, BEHAVIOR, AND COGNITION IN ADD
}

The effects of aspartame ( $34 \mathrm{mg} / \mathrm{kg} /$ day for 2 weeks) on the cognition, behavior, and monoamine metabolism of 15 children with a history of ADD were evaluated at the Yale University School of Medicine, using a randomized, double-blind, placebo-controlled crossover study design. Various measures including Conners Behavior ratings, Children's Checking Task, Airplane Test, and Wisconsin Card Sorting Test revealed no significant differences between aspartame and placebo. The Multigrade Inventory for Teachers showed a significant increase in activity level following aspartame treatments. Phenylalanine and tyrosine levels in plasma were significantly elevated at 1 and 2 hours after aspartame ingestion. (Shaywitz BA et al. Aspartame, behavior, and cognitive function in children with attention deficit disorder. Pediatrics Jan 1994;93:70-75). (Reprints: B A Shaywitz MD, Dept of Pediatrics, Yale University Sch of Med, New Haven, CT 06510).

COMMENT. The authors conclude from this study of 15 ADD children receiving single morning doses before school for 2 weeks that aspartame has no clinically significant effect on behavior and cognition, and does not affect urinary excretion rates of monoamines and metabolites. Studies of aspartame in children with neuropsychiatric problems are limited, but one well controlled evaluation in 10 children with absence seizures has shown that aspartame exacerbates EEG spike-wave discharges. (Camfield PR et al. Neurology 1992; 42:1000). The ingestion of aspartame in children with seizures should be limited or avoided until effects on seizure control are investigated further.(Ped Neur Briefs June 1992; $\underline{6}: 46-47)$. Migraine has been exacerbated by aspartame in controlled studies of adult patients.

\section{VASCULAR DISORDERS}

\section{STROKES AND VERTEBRAL ARTERY TRAUMA}

Three boys, ages 11,8 , and 7 years, with strokes from vertebral artery lesions resulting from neck trauma are reported from Indiana University School of Medicine, Indianapolis, and Baylor College of Medicine, Houston, TX. 